\title{
ABDOMINAL INJURY AND ITS OUTCOME- A PROSPECTIVE STUDY IN A TERTIARY HEALTH CARE CENTER
}

\author{
U. C. Singh ${ }^{1}$, Abhishek Jina ${ }^{2}$
}

1Professor, Department of General Surgery, BRD Medical College, Gorakhpur, Uttar Pradesh, India.

${ }^{2}$ Assistant Professor, Department of General Surgery, BRD Medical College, Gorakhpur, Uttar Pradesh, India.

ABSTRACT
BACKGROUND
In spite of the advancement in medical technology, trauma remains a major health threat causing significant mortality and
morbidity worldwide. The aim of this study was to evaluate the incidence and cause of the various abdominal injuries and compare
the incidence, management, and outcome of blunt trauma and penetrating trauma abdominal injuries.

\section{METHODS}

Total 99 patients who had visited the surgery department BRD Medical College, Gorakhpur, U. P., from April 2016 to March 2017 (one-year period) were included in this prospective study. Both blunt as well as penetrating trauma patients were included in the study. After recording a detailed history, all relevant laboratory and radiological investigations were done. Depending on the initial test reports, patients underwent either operative or conservative treatment. Postoperative complications, if any, were also recorded.

\section{RESULTS}

In the present study, majority of patients were in 11-20 years age group. Male to female ratio was found to be 5:1. Blunt trauma is more common than penetrating trauma with a ratio of 3.7:1, and in penetrating trauma stab injury is more common than gunshot injury with a ratio of 3:1. In blunt trauma abdomen, road traffic accident was the most common mode of injury (57.7\%) followed by falling from a height (30.8\%) and assault (10.2\%). In penetrating trauma abdomen, assault is the most common mode of injury (81\%) followed by a road traffic accident (9.5\%). Pain abdomen was the most common symptom (100\%) in both groups. Most of the patients were treated by conservative management in both groups. A low mortality rate was reported in the present study $(3.3 \%)$.

\section{CONCLUSIONS}

Abdominal trauma is one of the major health hazards among the younger male population. The most common mode of abdominal trauma is road traffic accident in the blunt trauma group, whereas in the penetrating group, gunshot and stabbing were the most common causes of injury. Conservative therapy was the most common mode of treatment in both groups. No major organ injuries were seen during radiological investigations.

HOW TO CITE THIS ARTICLE: Singh UC. Jina A. Abdominal injury and its outcome- a prospective study in a tertiary health care center. J. Evolution Med. Dent. Sci. 2019;8(18):1428-1432, DOI: 10.14260/jemds/2019/318

\section{BACKGROUND}

Worldwide, trauma continues to be a significant health hazard affecting an individual's life to a great extent. In every country across the world, maximum cases of trauma are associated with a high rate of morbidity and mortality. Regardless of the socioeconomic condition of the country, this health condition is reported to be the leading cause of hospitalization, and death in the first few decades of life.(1)

Abdominal trauma is frequently encountered by health professionals across the world and approximately one-third of the trauma patients were reported to have suffered from abdominal trauma.(2) The abdomen is a susceptible site for injury as it was a minimum of the bony structure surrounding it. The internal abdominal organs lay almost unprotected beneath the fat and muscle layer with any protection. Among

'Financial or Other Competing Interest': None.

Submission 13-03-2019, Peer Review 20-04-2019,

Acceptance 26-04-2019, Published 06-05-2019.

Corresponding Author:

Dr. Abhishek Jina,

Assistant Professor, Department of General Surgery,

27 Doctors Enclave, Rapti Nagar,

Phase III, Near Shahpur Thana,

Gorakhpur-27300, Uttar Pradesh, India.

E-mail: drabhishekjina@gmail.com

DOI: $10.14260 /$ jemds $/ 2019 / 318$ these abdominal trauma cases, $25 \%$ of cases required surgery.(3) The incidence of abdominal trauma is increasing day by day. In developing countries such as in India the urbanization, increased civic violence, road accidents, and criminal activities are mainly responsible for most of the abdominal injury cases. (4)

Usually, two forces are responsible for causing the injury to intrabdominal structures: compression forces and deceleration forces. The direct blow exerted by external forces gives rise to compression forces that can harm the hollow organs leading to its rupture. In deceleration forces, the linear shearing between the fixed and the free objects causes harm towards the solid viscera and causes a hematoma.(5)

Classically, abdominal injury can be divided into two types, either it is a blunt injury, or it can be a penetrating one. Penetrating trauma is usually diagnosed based on the clinical signs and symptoms, whereas, blunt trauma cases in a maximum of the instances get missed because of the presence of less obvious clinical signs. Among all abdominal trauma cases, $85 \%$ of the cases are blunt trauma cases.(6) Studies have shown that liver and spleen are the most common organs that get injured in the abdominal injury cases.(7)

Management of the abdominal trauma has several elements that start from pre-hospital care to the rapid transport of the patient to a trauma specialty center. The 
most important part of the management is the pre-hospital care that ultimately determines the fate of the patient. According to the guideline provided by the Advanced Trauma Life Support (ATLS) the most important part of the trauma care is Airway, Breathing, and Circulation or in short, this process is known as $\mathrm{ABC} .(8)$

However, due to the less clear understanding of the trauma treatment and support often these three basic steps are missed. In addition, in resource-poor countries such as India, where CT scan and other advanced technique such as Focused Assessment Sonography for Trauma or FAST are unavailable in the many tertiary healthcare set up, the diagnosis of trauma becomes difficult. These factors pose the major threats that affect the outcome of the trauma patients.(1)

In this context, this present study was undertaken to evaluate the impact of demographic damage on abdominal organs such as stomach, liver, spleen and large intestine and also to compare the diagnosis, management and the outcome of the blunt injury with the penetrating injury cases.

\section{METHODS}

This prospective study was undertaken on patients admitted in the surgical emergency department, BRD Medical College, Gorakhpur, U. P., with an abdominal injury, for a period of one year from April 2016 to March 2017. The patients were informed beforehand about the study and informed consent was signed. The prior approval from the ethics committee was obtained beforehand. After the patients were included in this study were monitored for various vitals including pulse rate, blood pressure, respiratory rate, and oxygen saturation.

After the initial history and clinical diagnosis was recorded general and systemic examination was conducted. A detailed laboratory investigation was conducted including haemoglobin, TLC, DLC, random blood sugar, PT, PC, INR, Serum urea, and Serum Creatinine. X-ray of the abdomen in $A P$ view, X-ray chest in PA view were done. In addition to this USG of the abdomen, and CECT of the abdomen was also performed depending on the requirement of the patients.

The decision of whether the patient will be kept under conservative treatment or he/she should be operated was taken after looking into the details of the laboratory investigation report and the hemodynamic status of patients. The patients who underwent operative procedures were monitored postoperatively for the following parameters-
- Pulse Rate, blood pressure, respiratory rate, temperature charting and input-output Charting.

- Abdominal Status was monitored by evaluating the bowel sound, tenderness, abdominal distension and passing of flatus and motion.

- Wound Examination

- Postoperative laboratory investigation including $\mathrm{Hb}$, TLC, DEC, Serum urea, Serum creatinine, random blood sugar, and Serum electrolyte e.g. Na, K, Ca.

Patients kept under the conservative treatment was monitored closely for any sign of deterioration and if the condition deteriorated then they were shifted for the operative intervention. The findings of the above parameters were tabulated, and the appropriate statistical analysis was done.

\section{RESULTS}

In the present study out of 99 patients with an abdominal injury, the maximum number of patients belong to the 11-20 years age group (25\%), followed by the 21-30 age group (22\%). Among these patients a maximum number of patients were male.

Out of a total of 99 patients, 78 patients had blunt trauma injury and 21 patients had a penetrating injury. Most of the abdominal injuries were due to road accidents (47\%). Among the blunt trauma group, $57.7 \%$ of injuries happened due to road traffic. In contrast, in the penetrating injury group, the road traffic and fall from height contributed an equal number of patients ( $9.5 \%$ of cases in each group).

Among the 99 patients, 12 were reported to have associated injuries such as chest trauma, head injury, perineal injury, and pelvic fracture. In the blunt injury as well the penetrating group the most common symptoms were a pain in the abdomen. On examination, tenderness was present in about 97 percent cases, guarding and rigidity in 59 percent cases and absent bowel sound was present in about 38.5 blunt trauma cases.

In blunt trauma abdomen patients, X-Ray abdomen shows normal findings in $56.4 \%$ cases compared to the $47.6 \%$ cases in the penetrating abdomen cases. In $15.4 \%$ of cases of blunt injury group, liver injury was noted in USG finding whereas in penetrating trauma cases no injury to Liver, Spleen and Urinary Bladder was reported.

\begin{tabular}{|c|c|}
\hline Age Group (Years) & Percentage of Patients \\
\hline $0-10$ & $14 \%$ \\
\hline $11-20$ & $25 \%$ \\
\hline $21-30$ & $22 \%$ \\
\hline $31-40$ & $18 \%$ \\
\hline $41-50$ & $11 \%$ \\
\hline $51-60$ & $5 \%$ \\
\hline$>60$ & $4 \%$ \\
\hline \multicolumn{2}{|c|}{ Table 1. Age Distribution of Patients } \\
\hline
\end{tabular}

\begin{tabular}{|c|c|c|c|}
\hline \multirow{2}{*}{ Type of Abdominal Injury } & \multirow{2}{*}{ Blunt Trauma Abdomen } & \multicolumn{2}{|c|}{ Penetrating Trauma Abdomen } \\
\cline { 3 - 4 } & & Stab & Gunshot \\
\hline No. of patients & 78 & 16 & 16 \\
\hline Percentage & 78 & \multicolumn{2}{|c|}{ Table 2. Type of Abdominal Injury } \\
\hline
\end{tabular}




\begin{tabular}{|c|c|c|c|c|c|c|}
\hline \multirow{2}{*}{ Mode of Injury } & \multirow{2}{*}{$\begin{array}{c}\text { No. of } \\
\text { Patients }\end{array}$} & \multirow{2}{*}{$\%$} & \multicolumn{2}{|c|}{ Blunt Trauma Injury Group } & \multicolumn{2}{|c|}{ Penetrating Abdominal Injury Group } \\
\hline & & & No. of Patients & $\%$ & No. of Patients & $\%$ \\
\hline Road traffic accident (RTA) & 47 & 47 & 45 & 57.7 & 2 & 9.5 \\
\hline Fall from height & 26 & 26 & 24 & 30.8 & 2 & 9.5 \\
\hline Assault & 25 & 25 & 8 & 10.2 & 17 & 81 \\
\hline Attacked by a wild animal & 1 & 1 & 1 & 1.2 & 0 & 0 \\
\hline \multicolumn{7}{|c|}{ Table 3. Mode of Injury } \\
\hline
\end{tabular}

\begin{tabular}{|c|c|c|c|c|c|}
\hline \multicolumn{2}{|c|}{ Penetrating Abdominal Injury Group } & \multicolumn{3}{c|}{ Blunt Trauma Injury Group } \\
\hline X-Ray Feature & $\begin{array}{c}\text { No. of } \\
\text { Patients }\end{array}$ & Percentage & X-Ray Feature & $\begin{array}{c}\text { No. of } \\
\text { Patients }\end{array}$ & Percentage \\
\hline Within normal limit (WNL) & 44 & 56.4 & Within normal limit(WNL) & 10 & 47.6 \\
\hline Gas under the diaphragm (good) & 23 & 29.5 & Gas under the diaphragm & 9 & 42.8 \\
\hline Ground glass appearance & 11 & 14.1 & Ground glass appearance & 2 & 9.5 \\
\hline Not done (ND) & 0 & 0 & Not done (nd) & 0 & 0 \\
\hline \multicolumn{7}{|c|}{ Table 4A. X-Ray Data } \\
\hline
\end{tabular}

\begin{tabular}{|c|c|c|c|c|c|}
\hline \multicolumn{2}{|c|}{ Penetrating Abdominal Injury Group } & \multicolumn{2}{c|}{ Blunt Trauma Injury Group } \\
\hline USG Findings & $\begin{array}{c}\text { No. of } \\
\text { Patients }\end{array}$ & Percentage & USG Findings & $\begin{array}{c}\text { No. of } \\
\text { Patients }\end{array}$ & \begin{tabular}{c} 
Percentage \\
\hline Intraperitoneal collection (IPC)
\end{tabular} \\
\hline Liver injury & 12 & 46.2 & Intraperitoneal collection (IPC) & 8 & 38 \\
\hline Splenic injury & $\mathbf{4}$ & 5.1 & Liver injury & 0 & 0 \\
\hline Renal injury & 3 & 3.8 & Splenic injury & 0 & 0 \\
\hline Bladder injury & 1 & 1.2 & Renal injury & 1 & 4.8 \\
\hline Partial wall hematoma & 1 & 1.2 & Partial wall hematoma & 0 & 0 \\
\hline Within normal limit (WNL) & 19 & 24.4 & Within normal limit (WNL) & 9 & 42.8 \\
\hline Not done (ND) & 16 & 20.5 & Not done (ND) & 4 & 19 \\
\hline
\end{tabular}

\begin{tabular}{|c|c|c|c|c|c|}
\hline \multicolumn{2}{|c|}{ Blunt Trauma Injury Group } & \multicolumn{2}{c|}{ Penetrating Abdominal Injury Group } \\
\hline Organ Injured & $\begin{array}{c}\text { No. of } \\
\text { Patients }\end{array}$ & Percentage & Organ Injured & No. of Pt. & Percentage \\
\hline Ileal perforation & 9 & 11.5 & Ileal perforation & 4 & 19.0 \\
\hline Jejunal perforation & 10 & 12.8 & Jejunal perforation & 4 & 19.0 \\
\hline Duodenal perforation & 2 & 2.6 & Duodenal perforation & 0 & 0 \\
\hline Transverse colon perforation & 0 & 0 & Transverse colon perforation & 1 & 4.7 \\
\hline Liver injury & 13 & 16.6 & Liver injury & 0 & 0 \\
\hline Splenic injury & 5 & 6.4 & Splenic injury & 0 & 0 \\
\hline Renal injury & 3 & 3.8 & Renal injury & 1 & 4.7 \\
\hline Bladder injury & 1 & 1.3 & Bladder injury & 0 & 0 \\
\hline Mesenteric tear & 12 & 15.4 & Mesenteric tear & 1 & 4.7 \\
\hline Omental tear & 0 & 0 & Omental tear & 1 & 4.7 \\
\hline None & 25 & 32.0 & None & 9 & 42.8 \\
\hline
\end{tabular}

\begin{tabular}{|c|c|c|}
\hline Hospital Stay in Days & No. of Patients & Percentage \\
\hline $0-5$ & 23 & 23 \\
\hline $6-10$ & 37 & 24 \\
\hline $11-15$ & 24 & 11 \\
\hline $16-20$ & 11 & 3 \\
\hline $21-25$ & 3 & 1 \\
\hline
\end{tabular}

\begin{tabular}{|c|c|c|}
\hline The Outcome of Abdominal Injury & No. of Patients & Percentage \\
\hline Improved and discharged & 88 & 88 \\
\hline Abscond & 6 & 6 \\
\hline Expired & 3 & 3 \\
\hline D.O.P.R. Table 5C. Outcome of Abdominal Injury & 2 \\
\hline \multicolumn{2}{|}{} \\
\hline
\end{tabular}




\section{DISCUSSION}

Abdominal trauma is a major health care threat that affects majorly the young population. Studies have shown that most of the young patients face abdominal trauma compared with their older counterparts. Manohar and Ramanaiah have reported $46 \%$ of the abdominal trauma patients in the 31 to 45 years of age group. In the present study, the incidence of trauma was more common in the 11-20 years of age group.(1)

In most of the studies reported a male predominance was found among the trauma patients. In a study by Abri et al among blunt abdominal trauma patients $63.9 \%$ were male.(8) The current study also has reported a higher percentage of males that get affected by the abdominal trauma compared to the females. This may be due to the reason that males are more involved with outdoor activity. In another study conducted in Karnataka, Maled and Todkar have shown that $82 \%$ of the abdominal trauma cases. $(9)$

The present study reported a higher number of blunt injuries $(n=78)$ compared to penetrating injuries $(n=21)$. Panchal et al have reported $74 \%$ of blunt injury cases. This result was quite similar to the present finding.(10) In contrast to the present finding Siddique et al have reported a higher number of penetrating trauma cases (64\%).(11) This difference in the finding of types of injury may be due to the sociodemographic and social influences where the study has been conducted. The majority of the injuries in the penetrating group was due to a gunshot or were stab injuries.

Extra-abdominal injuries are the most common complication that complicates treatment regimen and also the outcome measure of a trauma patient. studies have reported that the most common site of extra-abdominal injuries is head, neck and musculoskeletal injuries. ${ }^{(12,13)}$ In the present series, the extra abdominal injury was present in $12 \%$ cases. The most common extra abdominal injury was Chest trauma followed by Pelvic fracture and Head injury. In another study, $55.55 \%$ of extra-abdominal injuries were reported among trauma patients.(11) In the present study, abdominal pain was the most common presenting complaint accounting for $100 \%$ and abdominal tenderness was the most common sign accounting for $97.4 \%$ of cases.

Road accidents were the most common causes of injury in the blunt abdominal trauma group whereas; in the penetrating trauma group, $81 \%$ of the patients had assault as the main cause of injury. In a similar study conducted in Andhra Pradesh Manohar et al have shown that road accidents cause more abdominal injury among patients that were evaluated in their series.(1)

On further evaluation, it was reported that $32 \%$ of patients in the blunt trauma group had no organ injury compared to the $43 \%$ in the penetrating trauma group. The small intestine is the most common organ involved in blunt trauma group (24\%) followed by liver (16\%).

In the penetrating injury group, Jejunum and Ileum (Small intestine) perforations are the most common organ injury comprising of $38 \%$ cases. Transverse colon perforation, renal injury, mesenteric tear, and omental tear are least injured organs in the penetrating trauma group, each comprising $4.7 \%$ of cases. Maled and Todkar have reported a higher incidence of liver injuries (26\%) in their study followed by splenic injuries (17\%). ${ }^{(9)}$
Another study has reported a higher number of injuries were reported in the intestinal region for both penetrating and non-penetrating group. All the stomach injuries were reported in the penetrating trauma group. Kidney and splenic injuries were more common in the non-penetrating group.(11)

The mortality rate in the present study was $3.3 \%$, which is quite similar to the finding by the Abri et al,(8) Siddique et al,(11) Davis et al,(14) and Khan et al.(15) Mehta et al in a study conducted among blunt trauma patients reported a mortality rate of $4 \%{ }^{(3)}$

\section{CONCLUSIONS}

Abdominal trauma is one of the major health hazards among the younger male population. The most common mode of abdominal trauma is road traffic accident in the blunt trauma group, whereas in the penetrating group, gunshot and stabbing were the most common causes of injury. Conservative therapy was the most common mode of treatment in both groups. No major organ injuries were seen during radiological investigations.

\section{REFERENCES}

[1] Manohar K, Ramanaiah GV. Abdominal Trauma in adults - its outcome - a prospective study in a teritiary health care centre in Andhra Pradesh. Indian J Appl Res 2015;5(11):35-8.

[2] Sharma P, Agarwal A, Patel AH, et al. Management of cases of blunt trauma abdomen at the peripheral health care facility level - a suggested protocol. J Health Management 2016;18(1):182-7.

[3] Mehta N, Babu S, Venugopal K. An experience with blunt abdominal trauma: evaluation, management and outcome. Clin Pract 2014;4(2):599.

[4] Maske AN, Deshmukh SN. Traumatic abdominal injuries: our experience at the rural tertiary care center. Int Surg J 2016;3(2):543-8.

[5] Srivastava SK, Jaiswal AK, Kumar D. Prospective study of management and outcome of blunt abdominal trauma (solid organs and hollow viscus injuries). Int Surg J 2017;4(10):3262-71.

[6] Karamercan A, Yilmaz TU, Karamercan MA, et al. Blunt abdominal trauma: evaluation of diagnostic options and surgical outcomes. Ulus Travma Acil Cerrahi Derg 2008;14(3):205-10.

[7] van der Vlies CH, Olthof DC, Gaakeer M, et al. Changing patterns in diagnostic strategies and the treatment of blunt injury to solid abdominal organs. Int J Emerg Med 2011;4:47.

[8] Abri B, Vahdati SS, Paknezhad S, et al. Blunt abdominal trauma and organ damage and its prognosis. J Anal Res Clin Med 2016;4(4):228-32.

[9] Maled V, Todkar M. A profile of abdominal injuries in a tertiary care hospital. Indian J Forensic Med Toxicol 2017;11(2):169-72.

[10] Panchal HA, Ramanuj AM. The study of abdominal trauma: patterns of injury, clinical presentation, organ involvement and associated injury. Int Surg J 2016;3(3):1392-8.

[11] Siddique MAB, Rahman MK, Hannan A. Study of abdominal injury: an analysis of 50 cases. TAJ J Teach Assoc 2004;17(2):84-8. 
[12] Chalya PL, Mabula JB, Dass RM, et al. Injury characteristics and outcome of road traffic crash victims at Bugando Medical Centre in North western Tanzania. J Trauma Manag Outcomes 2012;6(1):1.

[13] Musau P, Jani PG, Owillah FA. Pattern and outcome of abdominal injuries at Kenyatta National Hospital, Nairobi. East Afr Med J 2006;83(1):37-48.
[14] Davis JJ, Cohn I, Nance FC. Diagnosis and management of blunt abdominal trauma. Ann Surg 1976;183(6):672-8.

[15] Khan WH, Mumtaz F, Farooka W. A Review of pattern of penetrating trauma in a surgical unit of mayo hospital, Lahore. Biomedica 2005;21:1-2. 